\section{EL USO DE TWITTER PARA MEJORAR LA COMPETENCIA DE LA EXPRESIÓN ESCRITA EN EL APRENDIZAJE DE LENGUAS EXTRANJERAS}

\author{
Germán Ruipérez \\ María Dolores Castrillo \\ José Carlos García Cabrero \\ Departamento de Filologías Extranjeras y sus Lingüisticas \\ Universidad Nacional de Educación a Distancia (UNED), \\ Madrid, España \\ lidil@vip.uned.es
}

\section{USING TWITTER TO IMPROVE WRITING COMPETENCE IN FOREIGN LANGUAGE LEARNING}

ABSTRACT: We present an empirical study on using Twitter as a tool for improving writing competence in distance learning German as a foreign language for students from the initial levels (A1 and A2 of the CEFR). It will describe the methodology and key findings of the fieldwork.

KEY WORDS: Distance learning; Twitter; web 2.0; collaborative learning; writing competence; German as a foreign language; DaF.

\section{INTRODUCCIÓN}

Tras impartir docencia a distancia de lenguas extranjeras durante más de veinte años, constatamos reiteradamente que es difícil motivar a los estudiantes de los niveles iniciales en la producción de textos en alemán, dado que el aprendizaje de la estructura oracional y del vocabulario les pueden resultar complejos, por lo que se producen errores recurrentes en los nativos hispanohablantes.

El objetivo principal de nuestro proyecto era precisamente mejorar la expresión escrita en alemán como lengua extranjera. Para ello, una de las mejores maneras era incentivar las producciones del alumnado intentando que superaran las barreras psicológicas iniciales, y que perdieran el miedo inicial a escribir.
RESUMEN: Se presenta un estudio empírico sobre la utilización de Twitter como herramienta para la mejora de la competencia de la expresión escrita en el aprendizaje a distancia de alemán como lengua extranjera de estudiantes de los niveles iniciales (A1 y A2 del MCER). Se describirá la metodología utilizada y las principales conclusiones del trabajo de campo.

PALABRAS CLAVE: Aprendizaje a distancia; Twitter; web 2.0; aprendizaje colaborativo; expresión escrita; alemán como lengua extranjera; DaF.

En este sentido, siempre hemos considerado los errores como oportunidades para aprender, y pensábamos que haciendo visibles estos errores comunes a través de sus propios escritos, los estudiantes podrían identificarlos fácilmente e intentarían evitarlos en el futuro, mejorando de esta forma la calidad de sus producciones.

Nuestra propuesta parte de la utilización de una herramienta informal y de un enfoque de aprendizaje colaborativo implementado mediante un sistema de corrección entre iguales, y se materializó como una parte de la evaluación continua en asignaturas de alemán de los niveles A1 y A2 (Marco Común Europeo de Referencia, MCER) de distintas titulaciones de Grado de la Universidad Nacional de Educación a Distancia (UNED) con sede en Madrid. Dichas asignaturas estaban encuadradas en el primer cuatrimestre de los planes de estudio correspondientes. 
De esta forma, incardinábamos con dos de los objetivos prioritarios en el desarrollo del Espacio Europeo de Educación Superior (EEES): mejorar (1) la competencia digital y (2) la competencia en una lengua extranjera. Se pretendia avanzar en el diseño de metodologías activas y materiales adecuados, para mejorar la calidad de la docencia y de la atención al estudiante, promoviendo así la autonomía del aprendizaje y la evaluación continua defendidas en el EEES.

\section{Diseño de LA ACTIVIDAD}

Según algunos autores, como Miguel Díaz (2006), las metodologías basadas en la resolución cooperativa de tareas o problemas se perfilan como paradigmas muy adecuados para la adquisición de competencias, pues entran en juego no solo las específicas y de conocimientos propios de la materia en cuestión, sino también las competencias genéricas transversales y las sistémicas.

Nuestra propuesta partía de principios pedagógicos constructivistas, en los que la base es "aprender haciendo" (learning by doing), de forma que el papel activo y la responsabilidad del aprendizaje recaen sobre el estudiante. Planteamos una revisión de las tareas que proponíamos hasta ahora a los estudiantes como parte de su evaluación continua: en lugar de pedirles la producción de una redacción de un determinado número de palabras sobre unos temas concretos -a partir de los contenidos tratados en los materiales didácticos-, decidimos introducir pasos intermedios que pudieran guiarles y motivarles a través de la consecución de metas más modestas, comenzando por oraciones sencillas para avanzar progresivamente hacia estructuras más complejas.

En consecuencia, buscábamos una metodología motivadora para los alumnos, y a la vez innovadora. Por este motivo, no nos pareció oportuna la utilización de herramientas formales, tales como los foros de debate del campus virtual de las asignaturas, sino que decidimos utilizar una herramienta más informal, y que pudiera permitir cierto anonimato si así se deseaba.

Por ello, optamos finalmente por utilizar Twitter por diferentes motivos: (1) limita las producciones a frases cortas (máximo 140 caracteres), (2) se puede hacer un fácil seguimiento a través de las etiquetas o hashtags, y (3), como muestran Haslam et al. (2009), no requiere especiales conocimientos técnicos, aparte de que (4) contribuye a la mejora de la competencia digital de los estudiantes, tal y como habían mostrado algunos estudios realizados preferentemente en Estados Unidos como el de Wankel (2011). En este sentido, aunque hasta la fecha se documentan escasos estudios empíricos relacionados con la utilización del microblogging como herramienta educativa, si se ha demostrado ampliamente la adecuación de otras similares como los blogs o wiki, tal y como recoge Blake (2008).

El último paso consistió en que fueran los propios estudiantes quienes seleccionaran los temas de interés sobre los que querían escribir, a partir de los campos semánticos tratados en el material didáctico. De esta forma, la motivación de los estudiantes se veía reforzada en tres aspectos: (1) escribian sobre los temas que ellos mismos habian elegido, (2) utilizaban un sistema de microblogging, novedoso en la metodología y en la tecnología, y (3) toda esta actividad se veía reflejada en la calificación de la asignatura como parte de la evaluación continua.

\section{Desarrollo del proyecto}

En primer lugar, y utilizando las herramientas de comunicación del curso virtual estándar proporcionado por la UNED, se comunicó a los alumnos la posibilidad de llevar a cabo la parte de evaluación continua de las asignaturas mediante una nueva tarea basada en Twitter. Básicamente se les informó sobre la repercusión en la calificación final y de las obligaciones que contraían al participar: (1) rellenar una encuesta inicial y otra final, (2) enviar un número mínimo semanal de tuits (el término inglés tweet se ha castellanizado frecuentemente como tuit), bien con aportaciones nuevas o bien corrigiendo alguna de los compañeros, y, por último, (3) realizar una redacción sobre el tema en cuestión a partir de la información de los tuits.

Dado lo novedoso de la propuesta, pensamos que era mejor contar únicamente con aquellos estudiantes que voluntariamente quisieran participar, dejando al resto las posibilidades de evaluación continua tradicionales. Por ello, el primer paso que tenían que dar quienes decidieran 
participar en el proyecto era rellenar la primera encuesta, con un doble propósito: (1) el equipo docente -y a la vez equipo investigador- dispondría de un perfil de los estudiantes especialmente sobre sus habilidades tecnológicas, y (2) los estudiantes definirian sus temas de interés a partir de las propuestas de contenidos tratadas en los materiales didácticos obligatorios.

\section{A. Encuesta inicial}

No sin cierta sorpresa, constatamos el éxito inicial de acogida de la iniciativa, pues 64 estudiantes (aproximadamente un $25 \%$ del total) se mostraron dispuestos a participar, a pesar de que la gran mayoría de ellos desconocian el uso de Twitter.

Entre los datos más significativos podemos destacar la presencia mayoritaria de mujeres (70,3\% frente a $29,7 \%$ de varones) de edades comprendidas principalmente entre 26 y 34 años (más del 45\%). Pensamos que es interesante explicitar también que tan solo un porcentaje bajo $(12,5 \%)$ eran menores de 25 años. Creemos que este hecho se debe al propio perfil de alumnado mayoritario en la UNED: adultos ya formados que buscan una segunda titulación. Estos porcentajes guardan aproximadamente la misma relación con respecto al total de alumnos matriculados en las asignaturas.

Prácticamente todos los participantes afirmaron tener conocimientos medios o altos de informática de usuario (correo electrónico, buscadores, redes sociales como Facebook, etc.). Sin embargo, solo 5 de los 64 participantes declararon utilizar Twitter con frecuencia, siendo elevadísimo el porcentaje $(82,8 \%)$ de personas que nunca antes habian utilizado esta herramienta, y solo un $18,2 \%$ se mostró al principio convencido de la utilidad de Twitter para mejorar su expresión escrita en alemán.

Por otra parte, entre los ocho temas propuestos, los tres más votados por los estudiantes fueron "Estilo de vida alemán", "Comida y recetas típicas" y "Aficiones".

\section{B. Formación guiada en Twitter}

El elevado desconocimiento del funcionamiento de Twitter por parte de los estudiantes nos obligó a ofrecerles una formación intensiva en dicha herramienta de microblogging.
A pesar de ser una tecnología relativamente sencilla (mensajes cortos que se publican secuencialmente en una línea temporal de más recientes a más antiguos) fue necesario enfatizar en los aspectos menos intuitivos, como por ejemplo, (1) las relaciones asimétricas de seguidor-seguido, (2) los hashtags para definir los temas y (3) poder hacer búsquedas globales.

Para hacer esta formación y llevar a cabo posteriormente el experimento, el equipo docente creó una cuenta específica en Twitter, @unedaleman. Cuando los participantes iban creando sus cuentas, se hacian seguidores nuestros y reciprocamente. De esta forma, nos asegurábamos de que hubiera una relación recíproca y simétrica de seguidoresseguidos, para que todos recibieran en sus líneas de tiempo los tuits del resto.

La formación inicial, llevada a cabo durante una semana, incluyó videotutoriales públicos en YouTube, tutoriales en formato electrónico, etc. y la resolución de dudas a través de otros canales tradicionales como correo electrónico, o los foros de debate de la plataforma institucional de la UNED. Se proporcionó también información sobre herramientas de escritorio $u$ online para gestionar eficazmente cuentas de Twitter como, por ejemplo, TweetDeck o HootSuite.

Pretendimos en todo momento que la formación fuera práctica, animando en todo momento a los estudiantes a empezar a escribir tuits de prueba, a utilizar hashtags, etc. a partir de unas actividades mínimas que el equipo investigador sugería, además de fomentar el uso de Twitter para resolver las dudas sobre la misma.

\section{Desarrollo de las actividades}

Una vez finalizadas las fases de inscripción y prácticas iniciales con Twitter, comenzaron las actividades propiamente dichas. Durante cuatro semanas los estudiantes tenían que enviar un mínimo de tres tuits a la semana, utilizando los siguientes hashtags para poder localizar rápidamente los tuits de un tema concreto: \#deutscheLebensart, \#Hobbys y \#kochenundessen.

Los mínimos inicialmente marcados, tres tuits semanales, fueron ampliamente superados por la mayoría de los participantes. Mientras que en las dos primeras semanas 
el equipo docente dejó fluir libremente las intervenciones para fomentar la participación y para que los estudiantes perdieran el miedo a escribir en alemán, en las dos últimas semanas se recomendó a los participantes no enviar nuevos tuits, sino intentar corregir los errores de alguno de los enviados anteriormente, con el fin de mejorar la calidad de las producciones escritas. Este segundo enfoque de corrección por pares dio lugar a la negociación de significados, a correcciones y a mejoras en la redacción a través del propio Twitter, y sirvió para que los propios estudiantes comenzaran a interiorizar cuáles eran sus errores más habituales al escribir alemán.

\section{Tarea y encuesta finales}

Después de la fase de generación de tuits, las tareas finales que tenían que llevar a cabo los estudiantes eran dos: (1) la elaboración de una redacción sobre uno de los temas propuestos a partir de la información de los tuits correspondientes, y (2) cumplimentar una encuesta final.

En lo que se refiere a la redacción, el propósito era intentar medir si la calidad de las producciones escritas era mejor que la de los estudiantes que no habían participado en el proyecto, fundamentalmente en lo que se refiere a la amplitud y corrección del vocabulario utilizado, y en la corrección de las estructuras sintácticas, prestando especial atención a los errores comunes sobre los que se había estado trabajando durante el mes de utilización de Twitter.

Aun teniendo en cuenta las limitaciones operativas y de representatividad del presente experimento, los resultados del estudio cualitativo de las redacciones fueron sorprendentemente muy positivos: (1) el número de errores era menor, y (2) los errores analizados habian disminuido notablemente, aparte de que (3) los estudiantes habian utilizado el vocabulario complementario adquirido durante la publicación de tuits.

Por otra parte, y aunque insistimos en que los datos deben ser valorados con cautela, para no extraer conclusiones precipitadas, los resultados de la encuesta final vinieron a reforzar (1) la tesis del aumento de la motivación para el aprendizaje y (2) la pertinencia del proyecto como forma de evaluación continua. Entre los principales datos positivos que podemos extraer de la encuesta final destacamos los siguientes:
1. El $77,7 \%$ de los participantes afirma que Twitter les gusta mucho. De hecho, tras finalizar este proyecto muchos de ellos continuaron utilizando Twitter como medio de comunicación personal, incluso redactando en alemán.

2. Entre las principales ventajas, los estudiantes destacan la capacidad de Twitter para mejorar la comunicación en alemán con los compañeros de asignatura $(83,3 \%)$ y con el equipo docente $(63,9 \%)$. Además, un grupo importante de ellos $(22,2 \%)$ confirmó que la limitación de 140 caracteres fue muy positiva, pues les resultaba más sencilla y menos intimidatoria a la hora de escribir sus primeras frases en la lengua meta. Aunque Twitter es una herramienta esencialmente asíncrona, el 55,6\% destacó la posibilidad de comunicación síncrona, es decir, que en muchas ocasiones intercambiaban mensajes continuamente como si se tratara de un chat.

3. El $72,2 \%$ está de acuerdo en que el uso de Twitter contribuyó significativamente a ampliar sus conocimientos de lengua alemana. El 66,7\% declara tener ahora más confianza para escribir en alemán, y el 74\% dice haber sido consciente de la importancia de la corrección por pares. Se valoran mucho los contenidos de actualidad y lo enriquecedor de la información proporcionada de primera mano por personas que residian en países de lengua alemana $(86,1 \%)$. La participación desinteresada de todos los miembros del grupo es muy valorada: el 76\% dijo haber disfrutado leyendo los tuits enviados por otros compañeros.

4. El $77,8 \%$ declaró que la tarea final en forma de redacción le resultó más atractiva y sencilla gracias a los tuits.

Por último, también queremos mencionar algunas objeciones expuestas por algunos estudiantes aislados, como por ejemplo las dificultades resultantes de tener que redactar textos de un máximo de 140 caracteres.

Además, se generó gran cantidad de información, superando ampliamente las expectativas iniciales (tanto por el número de participantes como por la cantidad de tuits generados), de tal manera que algunos estudiantes manifestaron que les resultó difícil asimilar tanta información y, del otro lado, un porcentaje significativo (38,9\%) mostró cierta adicción y ansiedad, con una atención continuada a la evolución de las publicaciones, etc. 
Entre las sugerencias recogidas figuran, por ejemplo, la eventual utilización en el futuro de herramientas similares, pero para la práctica de las destrezas orales en lugar de las escritas, o un calendario diferente, con más tiempo para la formación inicial en Twitter y mayor duración del período de tuits.

En resumen, las valoraciones subjetivas expresadas por los participantes en la encuesta final (con un elevado grado de satisfacción) y el análisis cualitativo de las producciones finales (que muestra un número significativamente menor de errores tipo) son muy positivos.

La respuesta a la última pregunta de la encuesta final viene a reflejar esta conclusión, además de la adecuación de Twitter y de las metodologías asociadas a estas tecnologías para llevar a cabo la experiencia propuesta: el 91,7\% de los participantes afirmó que volvería a participar en una tarea de aprendizaje basada en Twitter.

\section{Conclusiones}

Tras el estudio piloto realizado y descrito en el presente artículo, se sacaron las siguientes conclusiones provisionales sobre el uso de Twitter, que, como es natural, se pueden extrapolar a otras herramientas de microblogging, y que demuestran que Twitter puede ser utilizado como un recurso muy eficaz para mejorar el aprendizaje a distancia de la expresión escrita de una lengua extranjera, por los siguientes motivos:

1. Se favorece de manera notable la motivación del alumno en la producción propia de textos en una lengua extranjera, quien pierde muy rápidamente el miedo a redactar en una lengua diferente a la suya materna, gracias en parte a la obligada brevedad de sus mensajes (140 caracteres como máximo).

2. Con una metodología adecuada se puede mejorar cualitativamente la expresión escrita de cada estudiante en la lengua meta.

3. Estimula considerablemente la comunicación en una lengua extranjera entre los alumnos y con el equipo docente, aparte de que fomenta considerablemente la socialización, al reforzar la conciencia de grupo.

4. Frente a las reticencias mostradas inicialmente, después de llevar a cabo la tarea, Twitter es percibido por una gran mayoría del alumnado como una herramienta que favorece eficazmente su aprendizaje de la lengua extranjera.

Por ello, en un futuro serán verificadas y matizadas estas conclusiones en otros estudios empíricos ulteriores, con muestras mayores y más representativas, aparte de propuestas didácticas mejoradas, después de la experiencia acumulada en este primer proyecto piloto.

\section{BIBLIOGRAFÍA}

Blake, R. J. (2008): Brave new digital classroom, Georgetown University Press, Washington, DC.

Haslam, D. R.; Cornell, B. y Covalciuc, E. (2009): The Twitter Workbook, HMSI, inc., Plymouth.

Miguel Díaz, M. de (coord.) (2006): Metodologías de enseñanza y aprendizaje para el desarrollo de competencias. Orientaciones para el profesorado universitario ante el Espacio Europeo de Educación Superior, Alianza Editorial, Madrid.

Wankel, C. (2011): Educating Educators with Social Media (Cutting-Edge Technologies in Higher Education), Emerald Group Publishing, Bingley. 